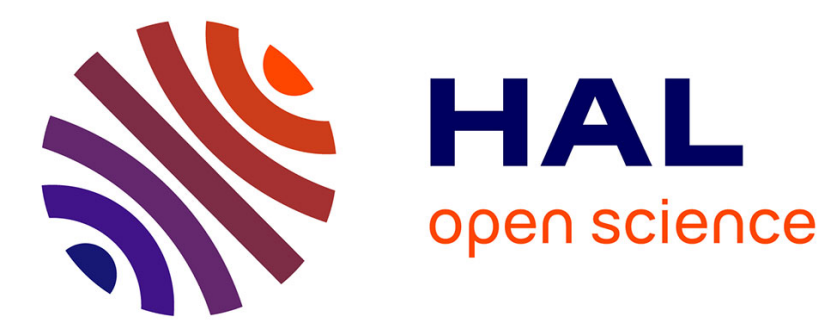

\title{
Epitaxial Growth of a Gold Shell on Intermetallic FeRh Nanocrystals
}

Patrizio Benzo, Ségolène Combettes, Cécile Garcia, Teresa Hungría, Béatrice Pécassou, Marie-José Casanove

\section{- To cite this version:}

Patrizio Benzo, Ségolène Combettes, Cécile Garcia, Teresa Hungría, Béatrice Pécassou, et al.. Epitaxial Growth of a Gold Shell on Intermetallic FeRh Nanocrystals. Crystal Growth \& Design, 2020, 10.1021/acs.cgd.0c00414 . hal-02617536

\section{HAL Id: hal-02617536 \\ https://hal.science/hal-02617536}

Submitted on 24 Aug 2020

HAL is a multi-disciplinary open access archive for the deposit and dissemination of scientific research documents, whether they are published or not. The documents may come from teaching and research institutions in France or abroad, or from public or private research centers.
L'archive ouverte pluridisciplinaire HAL, est destinée au dépôt et à la diffusion de documents scientifiques de niveau recherche, publiés ou non, émanant des établissements d'enseignement et de recherche français ou étrangers, des laboratoires publics ou privés. 


\title{
Epitaxial growth of a gold shell on intermetallic FeRh nanocrystals
}

\author{
Patrizio Benzo, ${ }^{* \dagger}$ Ségolène Combettes, ${ }^{\dagger}$ Cécile Garcia ${ }^{\dagger}$ Teresa Hungria, ${ }^{\ddagger}$ Béatrice \\ Pécassou, ${ }^{\dagger}$ and Marie-José Casanove ${ }^{*, \dagger}$ \\ †CEMES, CNRS UPR 8011 and Université de Toulouse, 29 rue Jeanne Marvig, F-31055 \\ Toulouse, France \\ $\ddagger$ Centre de MicroCaractérisation Raimond Castaing, Université de Toulouse, 3 rue \\ Caroline Aigle, F-31400 Toulouse, France \\ E-mail: patrizio.benzo@cemes.fr; casanove@cemes.fr
}

\begin{abstract}
In contrast with bimetallics, multimetallic nanoparticles (NPs) can combine different chemical orders in a same NP, which favors an enhanced tuning of their properties. In this work, trimetallic (Fe, Rh, Au) nanocrystals with controlled composition and chemical distribution were grown through a physical vapor deposition route using a two step process. First FeRh nanocrystals, $8.5 \mathrm{~nm}$ of mean diameter, were formed according to a Volmer-Weber growth mode. The growth conditions were tuned so as to achieve the atomic scale chemical order displayed by the intermetallic B2-FeRh phase. Then the gold layer was deposited at a lower temperature. Evidence is given for the complete coverage of the gold shell, which grows epitaxially over the B2-FeRh core exposed facets. These different features are particularly promising for further applications.
\end{abstract}




\section{Introduction}

Combining more than two different metals in a single nanoparticle (NP) offers new perspectives for generating novel physical and chemical properties. Such NPs are highly desirable for applications in many different fields including catalysis, plasmonics, electronics or magnetism. Presently a large effort is thus dedicated to new synthesis strategies for designing multimetallic NPs with controlled chemical composition. ${ }^{1}$ Beyond the composition, the distribution of the different elements inside the NP, i.e. forming alloys ${ }^{2,3}$ or separated domains $^{1,4-6}$ is also of the utmost importance for the properties of the NPs and their multifunctionality. For instance, high catalytic activity was combined with superior durability in $\mathrm{Au} @ \mathrm{FePt}_{3}$ core-shell NPs. ${ }^{4}$ The same three elements, with a different chemical distribution, were used to design FePt@Au core@shell NPs of interest for the biomedical field. ${ }^{5}$

Whereas a remarkable control of the NP composition has been achieved through efficient synthesis techniques, ${ }^{2,7,8}$ precise chemical distribution at the NP level remains difficult as it depends on many different factors. Already in bimetallic NPs, the large surface/volume ratio can promote the surface segregation of one of the elements of a binary alloy, which can lead to different geometries, among which a core-shell chemical distribution. ${ }^{9-13}$ The synthesis of intermetallics appears even more challenging at the nanoscale as chemically ordered alloys usually form at high temperature, not accessible to any synthesis route. Yet, intermetallic nanoparticles offer a better resistance to oxidation and display enhanced properties compared to disordered alloys. ${ }^{14,15}$

In this paper, we report the synthesis of trimetallic (Fe, Rh, Au) nanoparticles with wellcontrolled composition, crystalline quality and chemical distribution. These nanoparticles adopt a core-shell distribution, in which the chemically ordered intermetallic phase is

achieved in the FeRh alloy. In contrast with the Fe-Au and Rh-Au binary systems, ${ }^{16,17}$ which both display bulk-immiscibility, Fe and Rh are highly miscible in the whole range of composition and crystallize in a $\mathrm{CsCl}$ type intermetallic phase (B2 phase) close to the equiatomic composition. ${ }^{18}$ Among the three metals, Au has a much lower surface energy than the two 
others which, together with its larger size, is in favor of its segregation towards the NP surface. ${ }^{19}$ Consistently, gold rhodium nanoparticles were seen to display either Rh@Au core-shell geometry or ball-cup distribution with Rh partially encapsulated by Au. ${ }^{20,21} \mathrm{~A}$ core@shell distribution is also adopted in Fe@Au NPs. ${ }^{22-24}$ However, the synthesis way of the NPs, as the overall composition and environment can also lead to the formation of $\mathrm{RhAu}^{25}$ and $\mathrm{FeAu}^{26}$ alloys or Rh@Fe core-shell NPs. ${ }^{27}$ Thus, the precise chemical distribution in trimetallic (Fe,Rh,Au) NPs remains difficult to foresee and it will directly impact the properties of these NPs together with their potential applications in catalysis or in the biomedical field. Indeed, both $\mathrm{Au}$ and Rh are known for their catalytic properties and many gold or rhodium based bimetallic nanoparticles have demonstrated their enhanced performances compared to single metal NPs. ${ }^{28}$

There is also a great interest in alloying them with easily available low-cost metals as some 3d transition metals (Fe, Co or Ni for instance). ${ }^{26,29,30} \mathrm{Au}$ as well as $\mathrm{Rh}$, as recently demonstrated, ${ }^{31}$ are suitable for applications in the biomedical field. Substituting FeRh for Fe (or FePt) in core-shell Fe@Au (or FePt@Au) NPs can also be of great interest for this field. Besides, the intermetallic B2-FeRh phase is known to undergo a first order magnetic transition upon heating above $370 \mathrm{~K},{ }^{32,33}$ which is highly attractive for a wide range of applications $^{34,35}$ and its properties are known to be sensitive to both size and shape effects ${ }^{36,37}$ as well as to interface effects. ${ }^{38,39}$

Taking advantage of our expertise in the synthesis of B2-ordered FeRh nanoalloys ${ }^{36}$ and highly crystalline Fe@Au core-shell NPs ${ }^{22,24}$ using a magnetron sputtering technique, we were able to optimize the growth conditions to synthesize a homogeneous assembly of FeRh@Au core@shell trimetallic NPs in the $10 \mathrm{~nm}$ range. We report the atomic structure, chemical order and morphology observed in these NPs and compare them to related monometallic and bimetallic NPs. 


\section{Experimental details}

The trimetallic NPs were grown on amorphous alumina $\left(a-\mathrm{Al}_{2} \mathrm{O}_{3}\right)$ by dc magnetron sputtering from three high-purity Fe, Rh and Au elemental targets in an ultra-high vacuum (UHV) chamber. In order to facilitate transmission electron microscopy (TEM) observations, the alumina layer, about $5 \mathrm{~nm}$ thick, was itself deposited on a water soluble $\mathrm{NaCl}$ crystal. Both $\mathrm{NaCl}$ and alumina can stand the temperatures required to synthesize chemically ordered B2-FeRh. In order to achieve a core-shell distribution, we followed a two-step synthesis process. First, FeRh NPs were grown by co-deposition from Fe and Rh targets, in order to avoid the formation of small pure Rh NPs. A temperature of $600^{\circ} \mathrm{C}$ is sufficient to favor a Volmer-Weber growth mode of the FeRh NPs but a higher temperature, typically $750^{\circ} \mathrm{C}$ was necessary to achieve the desired intermetallic phase. The given temperatures are the ones measured at the rear of the substrate. Then the temperature was lowered to $600^{\circ} \mathrm{C}$ for

the deposition of the Au layer. The sputter conditions were tuned to near a 1:1:1 Fe:Rh:Au composition. Finally an amorphous alumina cover layer was deposited at room temperature to prevent water damage during the removal of the $\mathrm{NaCl}$ support. The $\mathrm{Au}$ layer deposition step was skipped in one of the samples in order to analyse the FeRh NPs before the growth of the $\mathrm{Au}$ shell.

The $\mathrm{NaCl}$ free films containing the trimetallic NPs were carefully deposited onto TEM copper grids to be studied through high-resolution TEM (HREM) and atomically resolved high-angle annular-dark-field scanning TEM (HAADF-STEM) experiments. These analyses were conducted using cold FEG Cs-corrected instruments. A Cs corrected Hitachi HF 3300C microscope was used for HREM studies and a probe corrected Jeol ARM200F microscope was used for HAADF-STEM and energy x-ray dispersive spectroscopy (EDS) studies. 


\section{Results and discussion}

\section{Forming B2- FeRh nanocrystals}

As reported in the experimental section, the FeRh cores were grown in the first step of the synthesis process. As observed in Figure 1, the FeRh NPs display a narrow size distribution with mean diameter around $8.5 \mathrm{~nm}$. The set of reflections displayed in the image Fourier transform, and better observed in its intensity profile (cf. Figure 1-c) is fully consistent with the B2-FeRh phase (CsCl type). The strong 100 reflection, forbidden in the body-centered cubic (bcc) lattice, originates from the B2 chemical order in which one element occupies the cube vertices and the other the cube center of the bcc unit cell, as displayed in the figure. When suitably oriented, typically along a [100] or a [110] zone axis, the two types of atomic planes (Fe rich and Rh rich ones) can be clearly distinguished in atomically resolved HAADF-STEM images, the rhodium atomic columns displaying a much brighter contrast than the iron ones. This contrast is clearly observed in Figure 1-d.

\section{From bi- to trimetallic NPs}

\section{Achieving a core-shell chemical distribution}

Gold was deposited in a second step after the substrate temperature was lowered to $600^{\circ} \mathrm{C}$. Figure 2 presents a general view of the NPs after gold deposition together with its corresponding size distribution histogram, which clearly stretches towards larger sizes. The important enlargement of the NPs can originate from the coalescence of several FeRh NPs. Indeed, the z-contrast HAADF/STEM image, displayed on the right side of the figure, shows that gold (in bright contrast) acts as a cement to assemble two or more FeRh NPS, leading to larger multicore NPs. Besides, the presence of a gold shell surrounding the FeRh NPs supports the hypothesis of a core-shell chemical distribution, also confirmed by the STEMEDS analysis displayed in Figure 3. The elemental maps presented in the figure clearly agree with a FeRh@Au core@shell distribution of the three elements, the FeRh alloy remaining in 


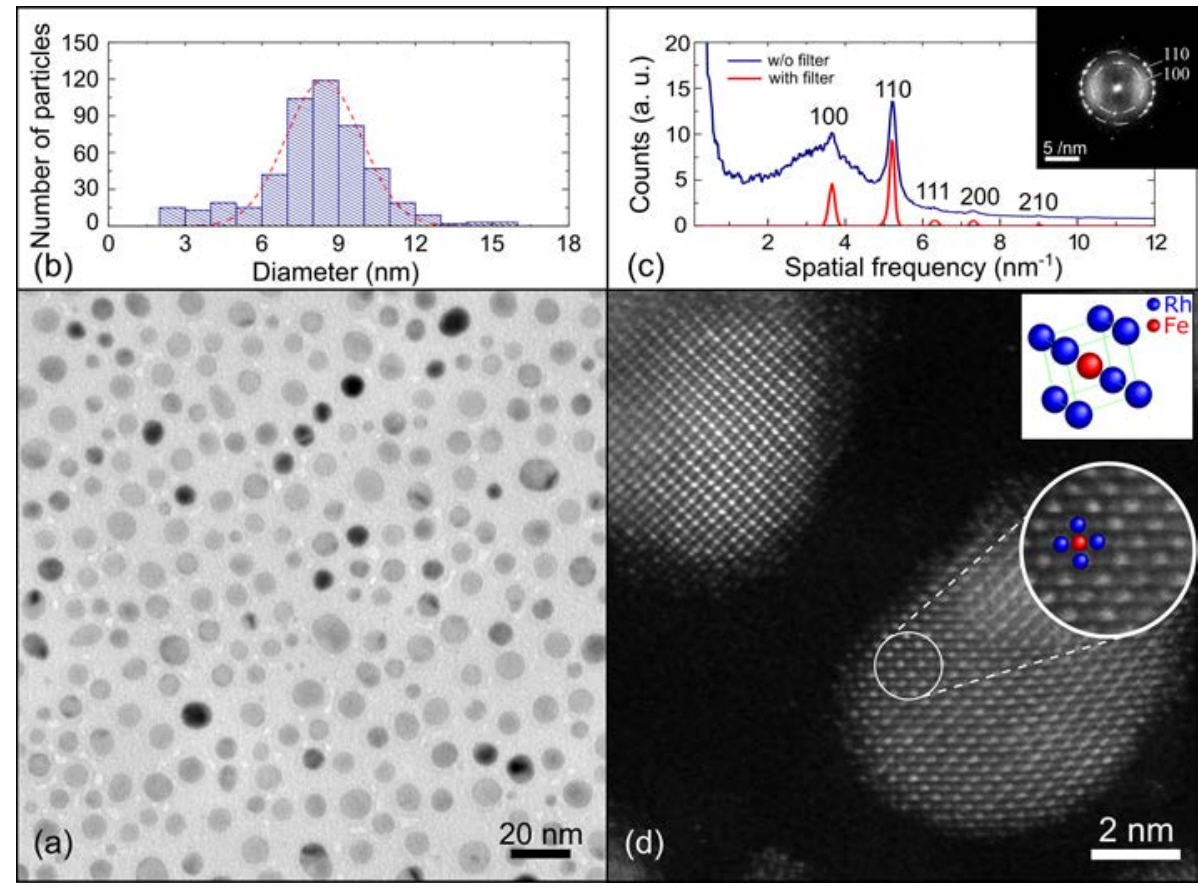

Figure 1: FeRh nanoparticles. (a) low magnification TEM image; b) associated size distribution histogram; c) intensity profile of the image Fourier transform displayed in the inset, with (blue) and without (red) the important background originating from the amorphous matrix; (d) atomically resolved HAADF-STEM image showing FeRh NPs observed along a $\langle 110\rangle$ direction for the NP at upper left and along a $\langle 100\rangle$ direction for the NP on the right. Both orientations are suitable for observing the B2 chemical order (the B2-FeRh unit cell is displayed at the upper right of the figure). As emphasized in the small enlarged region of the image, the bright large dots correspond to Rh atomic columns and the smaller ones to Fe columns. 


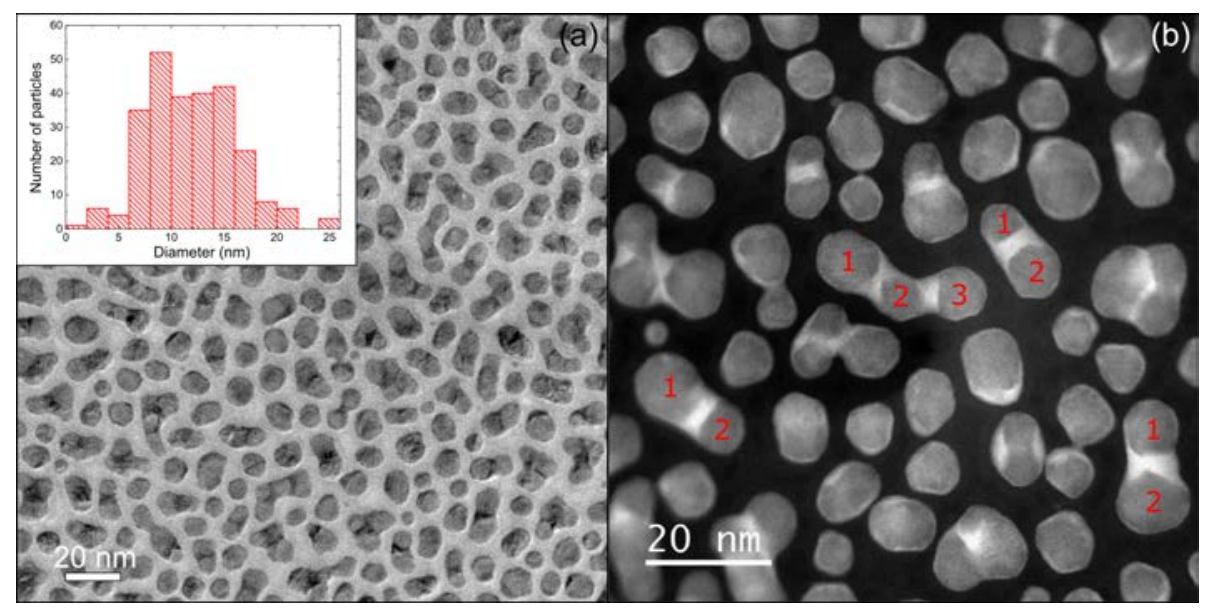

Figure 2: FeRh@Au nanoparticles. (a) TEM micrograph of the FeRh NPs after the deposition of a gold layer and its corresponding size histogram revealing an important enlargement of the NPs.(b) STEM-HAADF image of the trimetallic NPs showing the FeRh cores in gray and the gold regions in bright contrast. The image emphasizes the multicore nature of the larger nanoparticles (as the ones displayed with numbered cores).

the core of the NPs. Indeed, both Fe and Rh are present in all the NPs cores and no pure gold NPs are observed. This confirms that the gold deposition temperature promotes the diffusion of the Au atoms (or small clusters) on the alumina substrate towards FeRh NPs and not the growth of Au clusters.

\section{Epitaxial growth of the Au shell}

The structural analysis of FeRh@Au NPs oriented along a low-index zone axis (typically $\langle 100\rangle$ or $\langle 110\rangle$ FeRh lattice axes) shows that the B2-FeRh cores are mainly single crystals. The lattice parameter $a_{F e R h}=0.299 \pm 0.011 n m$, measured from the images is in good agreement with the lattice parameter reported in the literature for equiatomic B2-FeRh. ${ }^{18,40}$ Gold crystallizes in a face-centered cubic phase (fcc) with a lattice parameter $a_{A u}=0.40784$ nm. ${ }^{16}$ As previously observed in Fe@Au NPs, ${ }^{24}$ the crystalline gold shell grows along a $\langle 100\rangle_{f c c}$ direction on the $\{100\}_{B 2}$ FeRh facets and along a $\langle 111\rangle_{f c c}$ direction on the $\{110\}_{B 2}$ FeRh facets, the two main surface facets of the FeRh cores. Moreover, the quasi-continuity of the atomic planes across the different interfaces that can be observed in the NPs displayed 


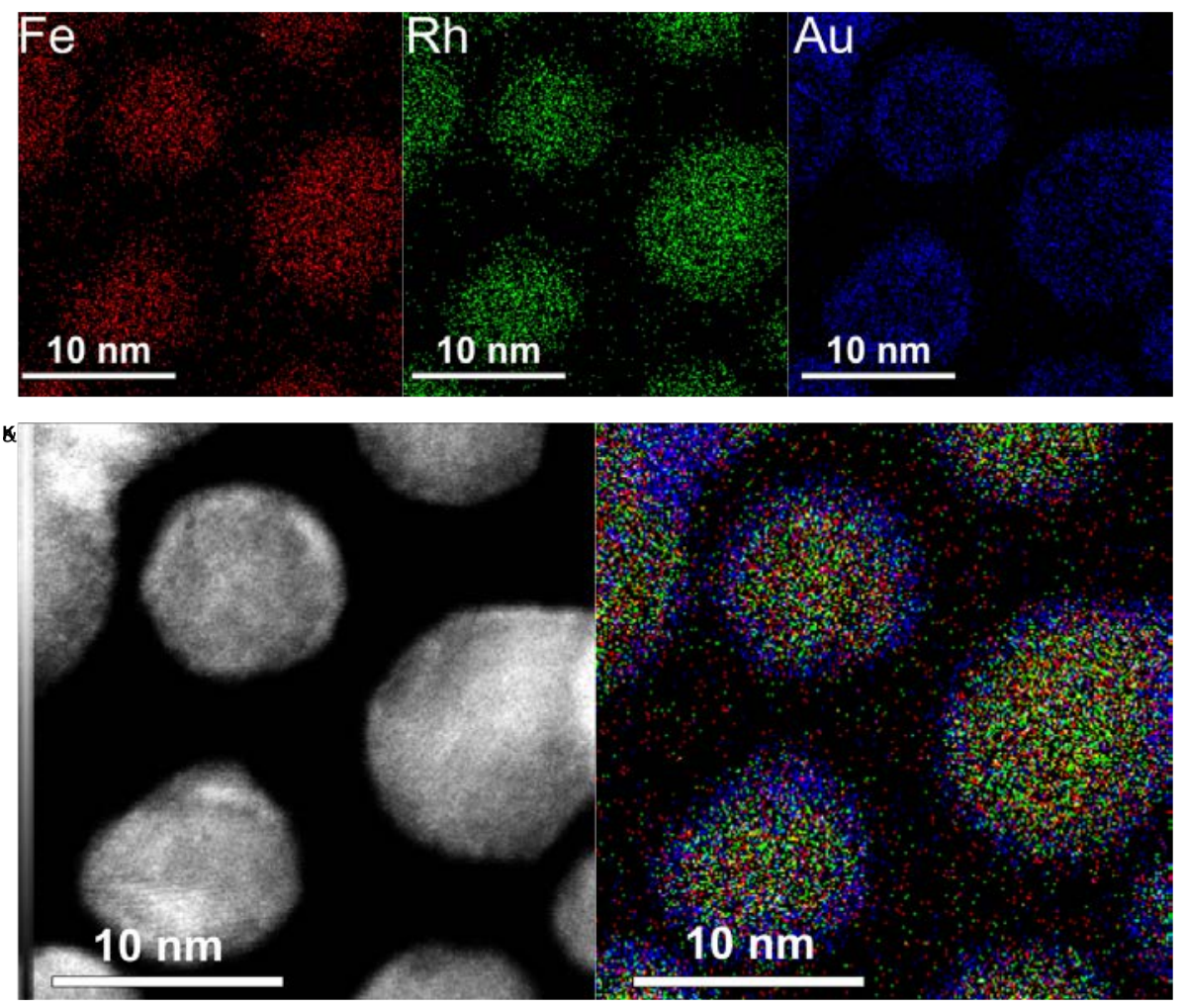

Figure 3: EDS analysis of the chemical distribution of the different elements. Top) Elemental maps of Fe, Rh and Au; bottom) HAADF-STEM image and corresponding chemical map emphasizing the FeRh-core@Au-shell distribution. 
in Figure 4, agrees with the occurrence of epitaxial growth. As the contrast displayed by HREM images is essentially phase contrast, hence sensitive to crystal structure and not to atomic number, the core-shell interface may be difficult to locate even in NPs observed along a low-index zone axis, depending on their precise orientation. This is typically the case for the NP displayed in figure 4-c. The artificial colors on the b and d images of figure 4 are aimed at improving the distinction between the fcc Au shell and the B2-FeRh core. The important misfit between the two different lattices, except at their $\{100\}_{f c c} /$ $\{100\}_{B 2}$ interface, is compensated by the introduction of misfit dislocations. Nevertheless, the NP displayed in figure 4-c,d exhibits a stepped interface on its right side, an unusual feature among the NPs that we observed. This feature may result from a different misfit accommodation mechanism as a stepped interphase boundary can be energetically favored. This should indeed be the case at the $\{111\}_{f c c} /\{110\}_{B 2}$ interface, which is expected to adopt a Kurdjumov-Sachs (KS) orientation owing to the ratio of the nearest neighbour distance in the two different phases (i.e. $0.288 \mathrm{~nm}$ in fcc $\mathrm{Au}$ and $0.259 \mathrm{~nm}$ in B2-FeRh, leading to a ratio equal to 1.11). ${ }^{41}$ However, the stepped interface displayed in this NP seems to include larger $\{100\}_{B 2}$ terraces than $\{110\}_{B 2}$ ones. We think that the occurrence of stepped interface may originate from the progressive reconstruction of the core-shell interface when the FeRh NP forming the core presents a large $\{110\}_{B 2}$ facet, which is scarsely observed. ${ }^{36}$ Besides, the nanoparticle displayed in figure 4-a,b presents a very regular shape in which the Au shell roughly reproduces the morphology adopted by the core. In contrast, the Au shell in figure 4-c,d clearly presents two hillocks on its right side. More generally, both centered core and off-centered core geometries are observed in the grown FeRh@Au NPs, contrary to what is observed in both Fe@Au NPs (only centered core) and Rh@Au ones (centered core or ball-cup). Most importantly, the Au shell always forms a complete coverage for the FeRh nanocrystals thus providing an efficient protection of the cores and preventing their coalescence. 


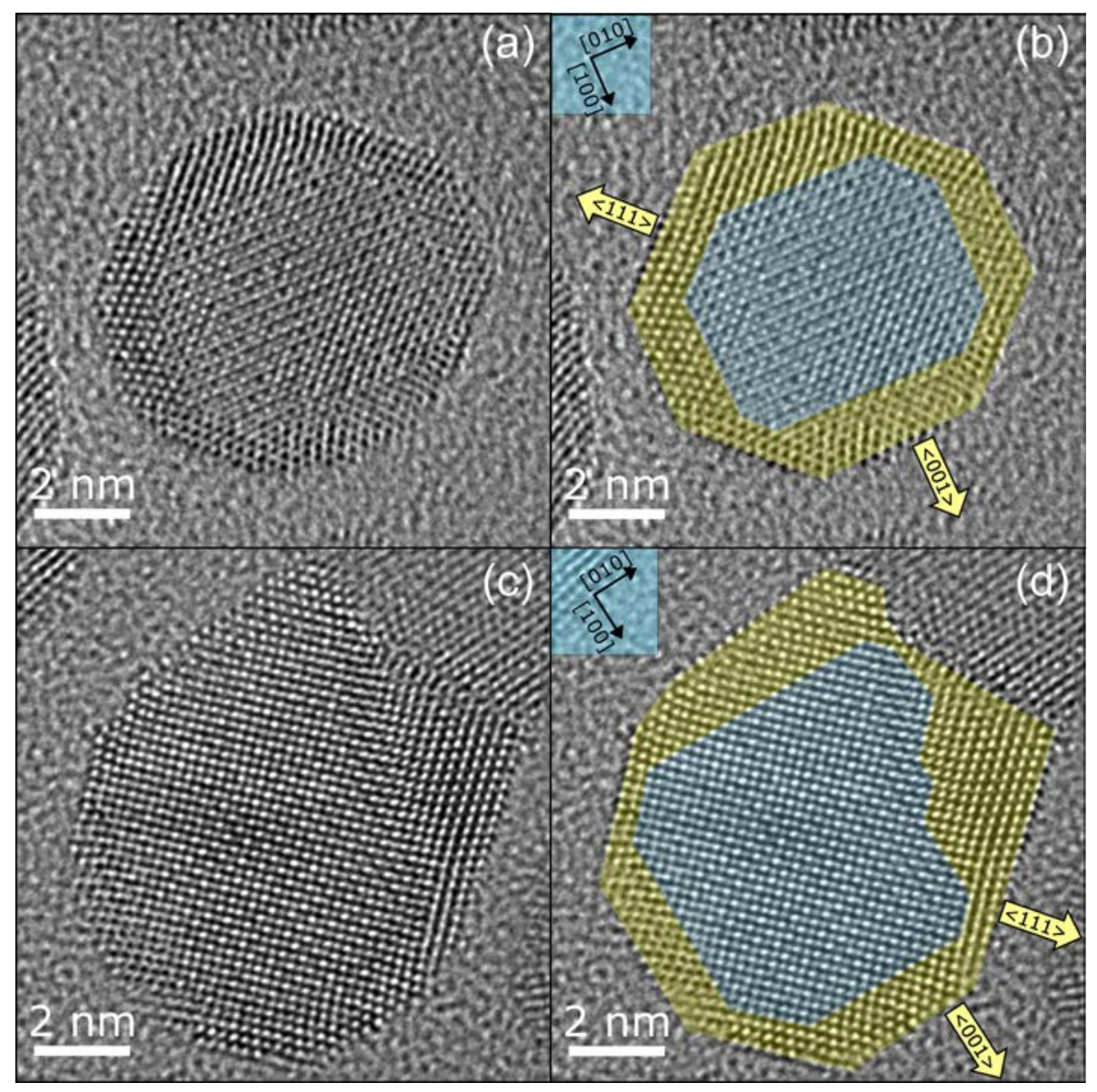

Figure 4: HREM images (greyscale and artificially coloured) of FeRh @ Au NPs: top) with regular shape; bottom) with off-centrered core. The orientations of the FeRh core and of Au shell, are indicated using the same colour as the corresponding regions. 


\section{Conclusion}

In conclusion, using a physical vapor deposition route, we succeeded in growing trimetallic (Fe, Rh, Au) nanoparticles in the $10 \mathrm{~nm}$ range with well controlled composition and chemical distribution. In particular, the FeRh core was successfully synthesized in its intermetallic B2 phase displaying a chemical order at the atomic level. Besides, the Au layer, deposited in the second step of the process, grows epitaxially on the $\{100\}_{B 2}$ and $\{110\}_{B 2}$ facets, which are the preferential surface facets of the nanocrystalline intermetallic core. As expected from thermodynamic considerations, Au not only remains at the surface of the FeRh crystals but also forms a complete shell, even if, in a large majority of the NPs, its thickness is not isotropic, leading to a NP geometry closer to Janus than to core-shell. ${ }^{9}$ Most importantly, together with this complete coverage, the low surface energy of the Au exposed facets, in particular the large $\{111\}_{A u}$ facets, should ensure the long term stability of the NPs geometry, making them highly suitable for the foreseen catalysis or biomedical applications.

\section{Acknowledgement}

This study was supported by the French National Research Agency (ANR) in the framework of the its "Investissements d'Avenir" program, ANR-10-LABX-0037-NEXT.

\section{References}

(1) Chen, P.-C.; Liu, X.; Hedrick, J. L.; Xie, Z.; Wang, S.; Lin, Q.-Y.; Hersam, M. C.; Dravid, V. P.; Mirkin, C. A. Polyelemental nanoparticle libraries. Science 2016, 352, $1565-1569$.

(2) Yao, Y.; Huang, Z.; Xie, P.; Lacey, S. D.; Jacob, R. J.; Xie, H.; Chen, F.; Nie, A.; $\mathrm{Pu}$, T.; Rehwoldt, M. Carbothermal shock synthesis of high-entropy-alloy nanoparticles. Science 2018, 359, 1489-1494. 
(3) Mattei, J.-G.; Grammatikopoulos, P.; Zhao, J.; Singh, V.; Vernieres, J.; Steinhauer, S.; Porkovich, A.; Danielson, E.; Nordlund, K.; Djurabekova, F.; Sowwan, M. Gas-Phase Synthesis of Trimetallic Nanoparticles. Chem. Mater. 2019, 31, 2151-2163.

(4) Wang, C.; van der Vliet, D.; More, K. L.; Zaluzec, N. J.; Peng, S.; Sun, S.; Daimon, H.; Wang, G.; Greeley, J.; Pearson, J.; Paulikas, A. P.; Karapetrov, G.; Strmcnik, D.; Markovic, N. M.; Stamenkovic, V. R. Multimetallic Au/FePt3 Nanoparticles as Highly Durable Electrocatalyst. Nano Lett. 2011, 11, 919-926.

(5) Yano, K.; Nandwana, V.; Chaubey, G. S.; Poudyal, N.; Kang, S.; Arami, H.; Griffis, J.; Liu, J. P. Synthesis and Characterization of Magnetic FePt/Au Core/Shell Nanoparticles. J. Phys. Chem. C 2009, 113, 13088-13091.

(6) Xu, Y.-H.; Wang, J.-P. FeCo-Au core-shell nanocrystals. Appl. Phys. Lett. 2007, 91, 233107.

(7) Lacey, S. D.; Dong, Q.; Huang, Z.; Luo, J.; Xie, H.; Lin, Z.; Kirsch, D. J.; Vattipalli, V.; Povinelli, C.; Fan, W.; Shahbazian-Yassar, R.; Wang, D.; Hu, L. Stable Multimetallic Nanoparticles for Oxygen Electrocatalysis. Nano Lett. 2019, 19, 5149-5158.

(8) Chen, P.-C.; Liu, G.; Zhou, Y.; Brown, K. A.; Chernyak, N.; Hedrick, J. L.; He, S.; Xie, Z.; Lin, Q.-Y.; Dravid, V. P.; O’Neill-Slawecki, S. A.; Mirkin, C. A. Tip-Directed Synthesis of Multimetallic Nanoparticles. J. Am. Chem. Soc. 2015, 137, 9167-9173.

(9) Ferrando, R.; Jellinek, J.; Johnston, R. Nanoalloys. From Theory to Applications of Alloy Clusters and Nanoparticles. Chem. Rev. 2008, 108, 845-910.

(10) Wang, L.-L.; Johnson, D. D. Predicted Trends of Core-Shell Preferences for 132 Late Transition-Metal Binary-Alloy Nanoparticles. J. Am. Chem. Soc. 2009, 131, 1402314029. 
(11) Fromen, M. C.; Morillo, J.; Casanove, M. J.; Lecante, P. Structure and chemical order in CoxRh1-x nanoparticles. Europhys. Lett. 2006, 73, 885-891.

(12) Bohra, M.; Grammatikopoulos, P.; Diaz, R. E.; Singh, V.; Zhao, J.; Bobo, J.-F.; Kuronen, A.; Djurabekova, F.; Nordlund, K.; Sowwan, M. Surface Segregation in ChromiumDoped NiCr Alloy Nanoparticles and Its Effect on Their Magnetic Behavior. Chem. Mater. 2015, 27, 3216-3225.

(13) Ferrando, R. Structure and Properties of Nanoalloys; Frontiers of Nanoscience; Elsevier, 2016; Vol. 10.

(14) Li, J.; Sun, S. Intermetallic Nanoparticles: Synthetic Control and Their Enhanced Electrocatalysis. Acc. Chem. Res. 2019, 52, 2015-2025.

(15) Wang, D.; Xin, H. L.; Hovden, R.; Wang, H.; Yu, Y.; Muller, D. A.; DiSalvo, F. J.; Abru ña, H. D. Structurally ordered intermetallic platinum-cobalt core-shell nanoparticles with enhanced activity and stability as oxygen reduction electrocatalysts. Nat. Mater. 2013, 12, 81-87.

(16) Okamoto, H.; Massalski, T.; Swartzendruber, L.; Beck, P. The Au-Fe (Gold-Iron) system. Bull. Alloy Phase Diagrams 1984, 5, 592-601.

(17) Okamoto, H.; Massalski, T. B. The Au-Rh (Gold-Rhodium) system. Bull. Alloy Phase Diagrams 1984, 5, 384-387.

(18) Swartzendruber, L. The Fe-Rh (Iron-Rhodium) system. Bull. Alloy Phase Diagrams $\mathbf{1 9 8 4 , ~ 5 , ~ 4 5 6 - 4 6 2 . ~}$

(19) Vitos, L.; Ruban, A.; Skriver, H.; Kollár, J. The surface energy of metals. Surf. Sci. 1998, 411, $186-202$.

(20) Piccolo, L.; Li, Z. Y.; Demiroglu, I.; Moyon, F.; Konuspayeva, Z.; Berhault, G.; Afanasiev, P.; Lefebvre, W.; Yuan, J.; Johnston, R. L. Understanding and control- 
ling the structure and segregation behaviour of AuRh nanocatalysts. Sci. Rep. 2016, 6,35226 .

(21) Demiroglu, I.; Fan, T.-E.; Li, Z. Y.; Yuan, J.; Liu, T.-D.; Piccolo, L.; Johnston, R. L. Modelling free and oxide-supported nanoalloy catalysts: comparison of bulk-immiscible Pd-Ir and Au-Rh systems and influence of a TiO2 support. Faraday Discuss. 2018, 208, $53-66$.

(22) Langlois, C.; Benzo, P.; Arenal, R.; Benoit, M.; Nicolai, J.; Combe, N.; Ponchet, A.; Casanove, M. J. Fully Crystalline Faceted Fe-Au Core-Shell Nanoparticles. Nano Lett. 2015, 15, 5075-5080.

(23) Kamp, M.; Tymoczko, A.; Schürmann, U.; Jakobi, J.; Rehbock, C.; Räzke, K.; Barcikowski, S.; Kienle, L. Temperature-Dependent Ultrastructure Transformation of AuFe Nanoparticles Investigated by in Situ Scanning Transmission Electron Microscopy. Cryst. Growth Des. 2018, 18, 5434-5440.

(24) Benzo, P.; Combettes, S.; Pecassou, B.; Combe, N.; Benoit, M.; Respaud, M.; Casanove, M. J. Role of the shell thickness in the core transformation of magnetic core(Fe)-shell(Au) nanoparticles. Phys. Rev. Mater. 2019, 3, 096001.

(25) Duan, Z.; Timoshenko, J.; Kunal, P.; House, S. D.; Wan, H.; Jarvis, K.; Bonifacio, C.; Yang, J. C.; Crooks, R. M.; Frenkel, A. I.; Humphrey, S. M.; Henkelman, G. Structural characterization of heterogeneous RhAu nanoparticles from a microwave-assisted synthesis. Nanoscale 2018, 10, 22520-22532.

(26) Vernieres, J.; Steinhauer, S.; Zhao, J.; Grammatikopoulos, P.; Ferrando, R.; Nordlund, K.; Djurabekova, F.; Sowwan, M. Site-Specific Wetting of Iron Nanocubes by Gold Atoms in Gas-Phase Synthesis. Adv. Sci. 2019, 6, 1900447.

(27) Ciuculescu, D.; Amiens, C.; Respaud, M.; Falqui, A.; Lecante, P.; Benfield, R. E.; 
Jiang, L.; Fauth, K.; Chaudret, B. One-Pot Synthesis of Core-Shell FeRh Nanoparticles. Chem. Mater. 2007, 19, 4624-4626.

(28) Louis, C. Chemical Preparation of Supported Bimetallic Catalysts. Gold-Based Bimetallic, a Case Study. Catalysts 2016, 6 .

(29) Soulé, J.-F.; Miyamura, H.; Kobayashi, S. Powerful Amide Synthesis from Alcohols and Amines under Aerobic Conditions Catalyzed by Gold or Gold/Iron, -Nickel or -Cobalt Nanoparticles. J. Am. Chem. Soc. 2011, 133, 18550-18553.

(30) Nakamula, I.; Yamanoi, Y.; Imaoka, T.; Yamamoto, K.; Nishihara, H. A Uniform Bimetallic Rhodium/Iron Nanoparticle Catalyst for the Hydrogenation of Olefins and Nitroarenes. Angew. Chem., Int. Ed. 2011, 50, 5830-5833.

(31) Kang, S.; Shin, W.; Choi, M.-H.; Ahn, M.; Kim, Y.-K.; Kim, S.; Min, D.-H.; Jang, H. Morphology-Controlled Synthesis of Rhodium Nanoparticles for Cancer Phototherapy. ACS Nano 2018, 12, 6997-7008.

(32) Fallot, M.; Hocart, R. Sur l'apparition du ferromagnetisme par elevation de temperature dans les alliages de fer et de rhodium (in french). Rev. Sci. 1939, 77, 498.

(33) Moruzzi, V. L.; Marcus, P. M. Antiferromagnetic-ferromagnetic transition in FeRh. Phys. Rev. B 1992, 46, 2864-2873.

(34) Cherifi, R. O. et al. Electric-field control of magnetic order above room temperature. Nat. Mater. 2014, 13, 345-351.

(35) Marti, X. et al. Room-temperature antiferromagnetic memory resistor. Nat. Mater. 2014, 13, 367-374.

(36) Liu, M.; Benzo, P.; Tang, H.; Castiella, M.; Warot-Fonrose, B.; Tarrat, N.; Gatel, C.; Respaud, M.; Morillo, J.; Casanove, M. J. Magnetism and morphology in faceted B2ordered FeRh nanoparticles. EPL 2016, 116. 
(37) Hillion, A.; Cavallin, A.; Vlaic, S.; Tamion, A.; Tournus, F.; Khadra, G.; Dreiser, J.; Piamonteze, C.; Nolting, F.; Rusponi, S.; Sato, K.; Konno, T. J.; Proux, O.; Dupuis, V.; Brune, H. Low Temperature Ferromagnetism in Chemically Ordered FeRh Nanocrystals. Phys. Rev. Lett. 2013, 110, 087207.

(38) Jekal, S.; Rhim, S. H.; Hong, S. C.; Son, W.-j.; Shick, A. B. Surface-terminationdependent magnetism and strong perpendicular magnetocrystalline anisotropy of an FeRh(001) thin film. Phys. Rev. B 2015, 92, 064410.

(39) Gatel, C.; Warot-Fonrose, B.; Biziere, N.; Rodríguez, L. A.; Reyes, D.; Cours, R.; Castiella, M.; Casanove, M. J. Inhomogeneous spatial distribution of the magnetic transition in an iron-rhodium thin film. Nat. Commun. 2017, 8, 15703.

(40) de Bergevin, F.; Muldawer, L. Crystallographic study of some iron-rhodium alloys (in french). C.R. Acad. Sci. 1961, 252, 1347-1349.

(41) van der Merwe, J.; Shiflet, G. The role of structural ledges at phase boundaries-III. F.C.C.-B.C.C. interfaces in Kurdjumov-Sachs orientation. Acta Metall. Mater. 1994, 42, $1199-1205$. 


\section{Graphical TOC Entry}

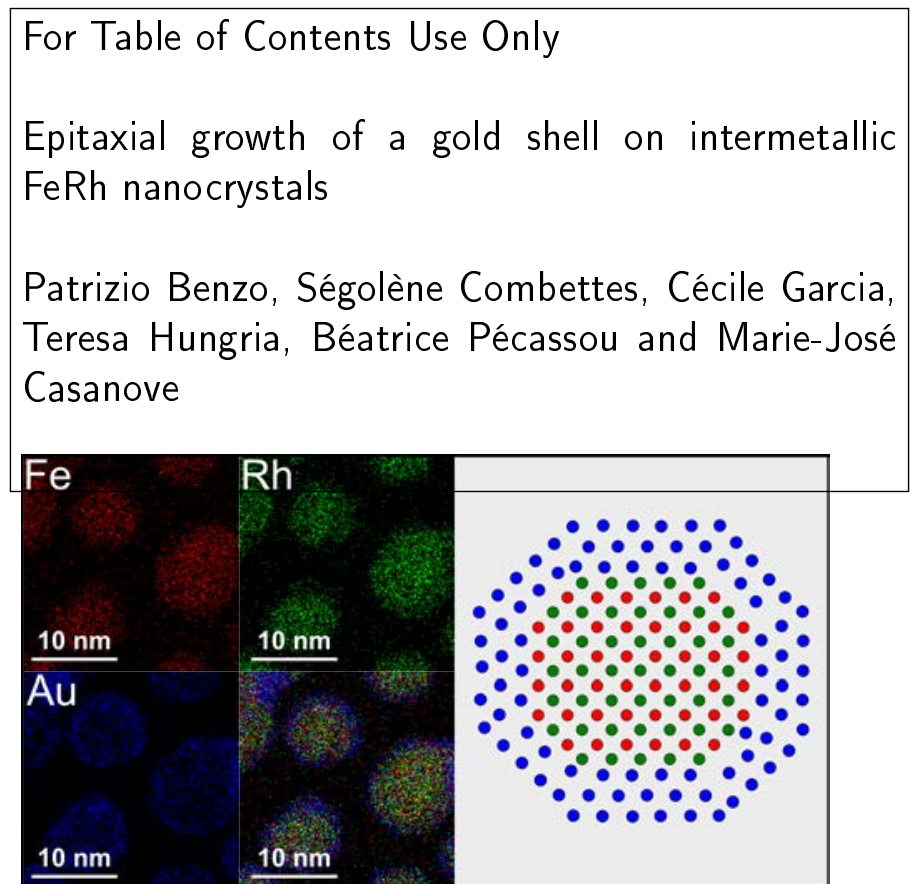

Synopsis: Precise chemical distribution of the elements is essential for a fine tuning of the properties of multimetallic nanoparticles. Using a physical route, trimetallic ( $\mathrm{Fe}, \mathrm{Rh}, \mathrm{Au})$ nanocrystals formed of intermetallic FeRh cores surrounded by $\mathrm{Au}$ shells were grown. Their structural features, as epitaxial coreshell interfaces, provide them with a highly stable geometry. 